\title{
Microarray expression profile analysis of circular RNAs and their potential regulatory role in bladder carcinoma
}

\author{
SHIJIE LI, YIQIAO ZHAO and XIAONAN CHEN \\ Department of Urology, Shengjing Hospital of China Medical University, Shenyang, Liaoning 110000, P.R. China
}

Received March 13, 2020; Accepted October 8, 2020

DOI: 10.3892/or.2020.7849

\begin{abstract}
Dysregulated circular RNAs (circRNAs) often contribute to the occurrence and development of various tumors; however, the function and mechanism of circRNAs are largely unknown in human bladder cancer (BC). In the present study, dysregulated circRNAs between BC and adjacent non-neoplastic bladder tissues were analyzed by circRNA microarray. We randomly selected 10 upregulated and five downregulated circRNAs for validation by quantitative real-time PCR. Bioinformatics analysis was further conducted to investigate the potential function of these differentially expressed circRNAs, with the differential expression of hsa_circRNA_100876, mir-136-5p, and mRNA-chromobox 4 (CBX4) subsequently verified. A total of 512 differentially expressed circRNAs were identified after scanning and normalization (340 upregulated and 172 downregulated circRNAs), with pathway and Gene Ontology analyses revealing their association with multiple significant cancer pathways. Construction of a circRNA-microRNA-mRNA network suggested additional potential roles of these circRNAs. The expression of hsa_circRNA_100876 and CBX4 was significantly negatively correlated with the expression of miR-136-5p. Additionally, hsa_circRNA_100876 was highly positively correlated with $\mathrm{CBX} 4$ expression. The results revealed that hsa_circRNA_100876 inhibition suppressed BC cell proliferation and it was associated with advanced $\mathrm{T}$ stage and lymphatic metastasis, and poor overall survival of BC patients. In conclusion, these differentially expressed circRNAs offer novel insights into potential biological markers or new therapeutic targets for the treatment of $\mathrm{BC}$. Furthermore, hsa_circRNA_100876 may increase the expression of CBX4 by competing with miR-136-5p, ultimately promoting the malignant biological behavior of BC. Aberrantly expressed hsa_circRNA_100876 could be used as a potential
\end{abstract}

Correspondence to: Dr Xiaonan Chen, Department of Urology, Shengjing Hospital of China Medical University, 36 Sanhao Street, Shenyang, Liaoning 110000, P.R. China

E-mail: chenxn@cmu.edu.cn

Key words: biomarker, circular RNAs, bladder cancer, microarray, prognosis non-invasive biomarker for the early detection and screening of $\mathrm{BC}$.

\section{Introduction}

Bladder cancer (BC) is among the most common malignant neoplasms of the urological and procreation system worldwide, with estimated new cases that exceed 380,000 and approximately 150,000 cancer-related deaths annually $(1,2)$. The occurrence of BC increases with age and is approximately 4-fold more prevalent in males than in females (3). Over $90 \%$ of BCs originate from the urothelial transitional epithelium, with $70 \%$ of patients presenting as non-muscle-invasive $\mathrm{BC}$ (NMIBC), which shows a tendency to recur but is generally not life threatening. Approximately $30 \%$ of patients are diagnosed with muscle-invasive BC (MIBC) at disease onset, with this diagnosis associated with high mortality due to recurrence or distant metastases. Furthermore, approximately $20 \%$ of NMIBC patients will undergo progression to MIBC, thereby worsening their prognosis (4). Early BC detection and intervention is important; therefore, a deeper understanding of the molecular changes in BC during tumorigenesis would help to develop a better therapeutic strategy for BC.

As a new regulatory molecule, non-coding RNA (ncRNA) represents an emerging player in numerous pathophysiological processes (5), including those involving tumor development, apoptosis, and proliferation (6). Circular RNA (circRNA) is an endogenous ncRNA that widely exists in the eukaryotic transcriptome $(7,8)$. CircRNAs are structured with a covalently closed loop via a back-splicing process through multiple mechanisms, which is different from linear RNAs harboring 5' caps and 3' tails $(9,10)$. CircRNAs were first identified as the result of spliceosome-mediated splicing errors (11); however, with the development of high-throughput sequencing and biotechnology, numerous functions of circRNAs have been recently revealed, including their roles in modulating gene expression, regulating alternative splicing, functioning as microRNA (miRNA) sponges or sequestering proteins (12-14).

Emerging evidence confirms that dysregulation of circRNAs is linked to several human diseases, including cancers. For instance, hsa_circ_0001445 has been revealed to be overexpressed in hepatocellular carcinoma and to play an important role in cell apoptosis, migration, and proliferation (15). Hsa_circ_0007835 has been revealed to represent a functional oncogene in tumorigenesis in lung cancer cells (16). 
In recent years, some researchers have investigated the role of aberrantly expressed circRNAs in the occurrence and development of BC $(17,18)$. However, the relevant in-depth research associated with the pathogenic mechanism of circRNAs remains in its infancy in BC.

In the present study, circRNA-expression profiles were screened and analyzed in three pairs of BC tissues and adjacent non-neoplastic bladder (ANNB) tissues through circRNA microarray, with the results identifying circRNAs with potential roles in $\mathrm{BC}$ tumorigenesis. Based on the profile, the competing endogenous RNA (ceRNA) networks in $\mathrm{BC}$ and the potential regulating relationships between the novel circRNA hsa_circRNA_100876, miR-136-5p and CBX4 were further investigated. Furthermore, bioinformatics analyses combined with experimental confirmation offered critical insight into the molecular signatures of circRNAs in $\mathrm{BC}$ carcinogenesis.

\section{Materials and methods}

Acquisition of tissue samples. Human BC tissues were selected from 43 patients (aged 50 to 68 years old) who received radical cystectomy between Oct 2015 and Dec 2017 at our institution (Shengjing Hospital of China Medical University, Shenyang, China) without preoperative neoadjuvant chemotherapy or radiotherapy. Three pairs of BC and matched ANNB tissues were used for circRNA microarray analysis, with another 40 pairs for subsequent validation. All samples were obtained within 5 min after resection and kept frozen in liquid nitrogen at $-80^{\circ} \mathrm{C}$. All tissue samples were pathologically evaluated by two well-experienced pathologists. The inclusion/exclusion criteria were as follows: i) pathologically confirmed bladder urothelial carcinoma; ii) no chemotherapy or radiotherapy before surgery; iii) no history of other malignant tumors; iv) complete clinicopathologic and follow-up data after surgery; and v) no evidence of distant metastasis at the time of surgery. All study participants provided their informed consent for inclusion in this study, and the protocol of the study was ratified and approved by the Research Ethics Committee of Shengjing Hospital of China Medical University (approval no. 2017PS012J).

CircRNA microarray hybridization. A NanoDrop ND-1000 (Thermo Fisher Scientific, Inc.) was used to quantify total RNA from each sample. Briefly, circRNAs were enriched by eliminating linear RNAs with Rnase R (Epicentre Biotechnologies), followed by amplification and transcription into fluorescent circRNA using a random priming method (Arraystar Super RNA labeling kit; Arraystar, Inc.). The labeled cRNAs were hybridized onto the Arraystar Human circRNA Array V2 (8x15K; Arraystar, Inc.). After washing the slides, the hybridized arrays were washed by Arraystar Circular RNA microarray v 2.0. and then fixed on the chip scanner for signal value detection. Then, $1 \mu \mathrm{g}$ of each labeled cRNA was fragmented by adding $5 \mu \mathrm{l} 10 \mathrm{X}$ Blocking Agent and $1 \mu \mathrm{l}$ of $25 \mathrm{X}$ fragmentation buffer, then heated the mixture at $60^{\circ} \mathrm{C}$ for $30 \mathrm{~min}$. Subsequently, $25 \mu \mathrm{l}$ of $2 \mathrm{X}$ hybridization buffer was added to dilute the labeled cRNA. Then, $50 \mu 1$ of hybridization solution was dispensed into the gasket slide and assembled to the circRNA expression microarray slide.
Finally, hybridized arrays were scanned using an Agilent Scanner G2505C (Agilent Technologies, Inc.).

Microarray data analysis. The feature extraction software (v11.0.1.1; Agilent Technology, Inc.) was use to analyze the collected array images. The limma package from the R software package (https://www.r-project.org/) was used for quantile normalization and subsequent data processing. CircRNA exhibiting fold change (FC) $\geq 2$ and a $\mathrm{P}<0.05$ were considered significant. Volcano-plot (generated by R Software R-3.3.1 gplots) was performed to identify differentially expressed circRNAs with statistical significance between two groups and fold-change filtering was performed to identify differentially expressed circRNAs between two groups. Hierarchical clustering (produced by R software R-3.3.1 gplots, function heatmap2) was performed to display variable circRNA-expression patterns among samples. Data conversion is based on Z-Score. Arraystar miRNA target-prediction software utilizing the TargetScan (http://www.targetscan.org/) (19) and miRanda (http://www.miranda.org) (20) databases was used to investigate circRNA-miRNA relationships, with the top five predicted miRNAs for each differentially expressed circRNA extracted for further analysis. CircRNA-miRNA interactions were annotated in detail.

Reverse transcription-quantitative PCR (RT-qPCR) validation. In order to be more representative of the chip as a whole, 40 sets of BC and ANNB tissues were randomly selected from the microarray for further verification by RT-qPCR analysis. Total RNA was extracted from each frozen sample (the amount of tissue was $\sim 100 \mathrm{mg}$ ) in accordance with the manufacturer's protocol (RNAiso PLUS; Takara Bio, Inc.). The $1 \%$ agarose electrophoresis determined the integrity of the isolated RNA, and PrimeScript RT reagent kit with gDNA Eraser (Takara Bio, Inc.) was used to synthesize first-strand cDNA. A spectrophotometer was used to measure the optical density ratio at 260 and $280 \mathrm{~nm}(\mathrm{OD} 260 / 280=1.8-2.0)$ to determine RNA concentration. Primer 5.0 software (Premier Biosoft) was used to design specific primers, which are listed in Table SI. A StepOnePlus real-time PCR system with SYBR-Green I (Takara Bio, Inc.) was used to conduct RT-qPCR. Glyceraldehyde 3-phosphate dehydrogenase (GAPDH) was used as an internal control. Each sample was tested in triplicate. The thermocycling conditions used were as follows: $95^{\circ} \mathrm{C}$ for $30 \mathrm{sec}$, followed by 40 cycles at $95^{\circ} \mathrm{C}$ for $5 \mathrm{sec}$ and $60^{\circ} \mathrm{C}$ for $30 \mathrm{sec}$. Relative mRNA expression levels were determined using the $2^{-\Delta \Delta \mathrm{Cq}}$ method (21).

Dual luciferase reporter assay. The mutant and wild-type sequences of hsa-circRNA-100876 were cloned downstream of the firefly luciferase gene pGL3 vector (Promega Corporation). Based on the instructions of the manufacturer, Luciferase Reporter plasmid (Promega Corporation) and miR-136-5p expression plasmid (pmirGLO) were co-transfected instantaneously utilizing Lipofectamine 2000 (Invitrogen; Thermo Fisher Scientific, Inc.). Subsequently, pGL3-hsa_circRNA_100876 vectors and miR-136-5p mimics along with negative control were co-transfected into 293 cells (Bena Culture Collection). Then, 48 h later, Dual-Luciferase reporter gene assay kit (Promega Corporation) was used 
to detect luciferase activity. The sequences of miR-136-5p mimics were provided by RiboBio Biotechnology Co., Ltd. and were as follows: forward, 5'-ACUCCAUUUGUUUUG AUGAUGGA-3' and reverse, 5'-UCCAUCAUCAAAACA AAUGGAGU-3'. For each analysis, the Renilla luciferase signal was standardized to the firefly luciferase signal.

Cell culture and CCK-8 assay. The BC cell lines EJ-1 (EJ) and T24 used in the present study were obtained from the Cell Bank of the Chinese Academy of Sciences in Shanghai. In addition, both cells lines were authenticated by STR profiles. Cells were grown at $37^{\circ} \mathrm{C}$ in a humidified atmosphere of $5 \% \mathrm{CO}_{2}$. All the cell lines were cultured in DMEM medium (Invitrogen; Thermo Fisher Scientific, Inc.) supplemented with $1 \%$ penicillin-streptomycin and $10 \%$ fetal bovine serum (FBS; Gibco; Thermo Fisher Scientific, Inc.). Whether hsa_circRNA_100876 is involved in $\mathrm{BC}$ cell proliferation ability was measured using Cell Counting Kit-8 (CCK-8; Dojindo Molecular technologies, Inc.) assay. Cells in each group were digested by $0.25 \%$ trypsin and seeded into 96-well plates (5,000 cells/well); and three duplicate wells were set for each group of cells. CCK-8 $(10 \mu \mathrm{l})$ reagent was added to each well and incubated at $37^{\circ} \mathrm{C}$ for $150 \mathrm{~min}$ after transfection for $24,48,72$ and $96 \mathrm{~h}$. All the experiments were performed in triplicate. The absorbance was detected at $450 \mathrm{~nm}$ using a microplate reader instrument (Sunrise ${ }^{\mathrm{TM}}$; Tecan Group, Ltd.). The ratio of absorbance difference between the experimental group and blank control group/24-h difference was the cell proliferation rate. The small interfering RNA (siRNA) targeting circRNA_100876 (si-circRNA_100876) was provided by RiboBio Biotechnology Co., Ltd. and was as follows: si-circRNA_100876, 5'-CTCCTACAATGTTGATATG-3'. si-NC (product no. siN0000001-1-5) was also provided by RiboBio Biotechnology Co., Ltd.

Bioinformatics analysis. Gene Ontology (GO) enrichment analysis (22) was performed to determine functional annotations of miRNA target genes, mainly including three independent ontologies (molecular function, cellular component and biological process). TopGO (https://bioconductor. org/packages/release/bioc/html/topGO.html; version 2.32.0) was used to perform $\mathrm{GO}$ analysis of the differentially expressed genes to infer their involvement in molecular functions. Kyoto Encyclopedia of Genes and Genomes (KEGG) enrichment analysis (23) was used to clarify the interactions and functions among these differentially expressed genes. The $\mathrm{R}$ script was used to calculate the significance of differentially expressed genes and KEGG through hypergeometric distribution, and Fisher's exact test was used to calculate the P-value. Data downloads were based on the GO website (http://geneontology. org) and KEGG website (https://www.genome.jp/kegg/). The threshold for the P-value was $<0.05$ and the count number $>2$. The top 10 enriched GO items and KEGG pathways of the differentially expressed mRNAs were ranked by enrichment score $[-\log 10$ (P-value)].

Construction of a circRNA-miRNA-mRNA regulatory network. The selected significantly expressed circRNAs, predicted miRNAs and protein-coding mRNAs were used to construct a circRNA-miRNA-mRNA regulatory network by a software based on TargetScan and miRanda (http://www.miranda.org). The software that was used considered the capacity and number of circRNA-microRNA binding sites, as well as the binding capacity of microRNA and mRNA.

Western blotting. Total proteins ( $100 \mathrm{mg}$ of tissue) were extracted from cell lysates with wash buffer (1X PBS, $0.1 \%$ SDS, $0.5 \%$ nonidet-P-40 and $0.5 \%$ sodium deoxycholate) containing $1 \%(\mathrm{w} / \mathrm{v})$ protease inhibitor (Beyotime Institute of Biotechnology), and a BCA protein assay kit (Beyotime Institute of Biotechnology) was used to quantify the protein concentrations. Protein extract $(80 \mu \mathrm{g})$ was loaded onto $10 \%$ SDS-PAGE gels and then transferred to polyvinylidene fluoride membranes (EMD Millipore). The membranes were then incubated with Tris-buffered saline containing $0.05 \%$ Tween-20 and 5\% skim milk powder for $2 \mathrm{~h}$ at room temperature in the presence of the rabbit anti-CBX4 monoclonal antibody at $4^{\circ} \mathrm{C}$ overnight (dilution 1:1,000; cat. no. PA5-109482; Thermo Fisher Scientific, Inc.), followed by incubation for $120 \mathrm{~min}$ at room temperature with the corresponding secondary antibody HRP-conjugated Affinipure goat anti-rabbit $\operatorname{IgG}(\mathrm{H}+\mathrm{L})$ (dilution 1:2,000; cat. no. SA00001-2, ProteinTech Group, Inc). GAPDH was used as an internal reference (1:10,000; cat. no. 10494-1-AP; ProteinTech Group, Inc.), and an enhanced chemiluminescence kit was used for visualization (Beyotime Institute of Biotechnology). The relative expression of the target protein was quantified by ImageJ software (version $1.48 \mathrm{v}$; National Institutes of Health).

Immunohistochemical staining. The 40 pairs of paraffin sections from BC tissues and ANNB tissues were prepared and analyzed. The sections (4- $\mu \mathrm{m}$ thick) were fixed with $10 \%$ neutral buffer formalin at room temperature for $12 \mathrm{~h}$. The sections were randomly numbered and double blinded. Immunohistochemistry was performed using an SP kit (ZSGB-BIO; OriGene Technologies, Inc.). After rinsing with phosphate-buffered saline (PBS), $3 \% \mathrm{H}_{2} \mathrm{O}_{2}$ was added at room temperature for $15 \mathrm{~min}$. Then the sections were sealed with goat serum at room temperature for $15 \mathrm{~min}$ (ZSGB-BIO; OriGene Technologies, Inc), followed by antigen retrieval in a microwave (total power $800 \mathrm{~W}$, time $10 \mathrm{~min}$ ). An appropriate dilution of primary antibody (CBX4; 1:200; cat. no. sc-517216; Santa Cruz Biotechnology, Inc.) was then added and the sections were incubated overnight in wet box at $4^{\circ} \mathrm{C}$. The sections were then washed and incubated for $30 \mathrm{~min}$ at $37^{\circ} \mathrm{C}$ with a secondary antibody [HRP-conjugated Affinipure goat anti-mouse IgG $(\mathrm{H}+\mathrm{L})$; cat. no. SA00001-1; ProteinTech Group, Inc], rinsed with PBS and then incubated with horseradish oxidase-labeled streptomycin avidin working solution (ZSGB-BIO; OriGene Technologies, Inc.) for $30 \mathrm{~min}$ at $37^{\circ} \mathrm{C}$. Sections were then rinsed again in PBS and antibody binding was developed using a DAB chromogenic kit (Beyotime Institute of Biotechnology). Sections were then counterstained with hematoxylin at room temperature for $90 \mathrm{sec}$. A light microscope (original magnification, x100; Nikon Corporation) was used to score the results of immunostaining, by multiplying the score of staining intensity of positive cells and the score of the percentage of positive cells. 

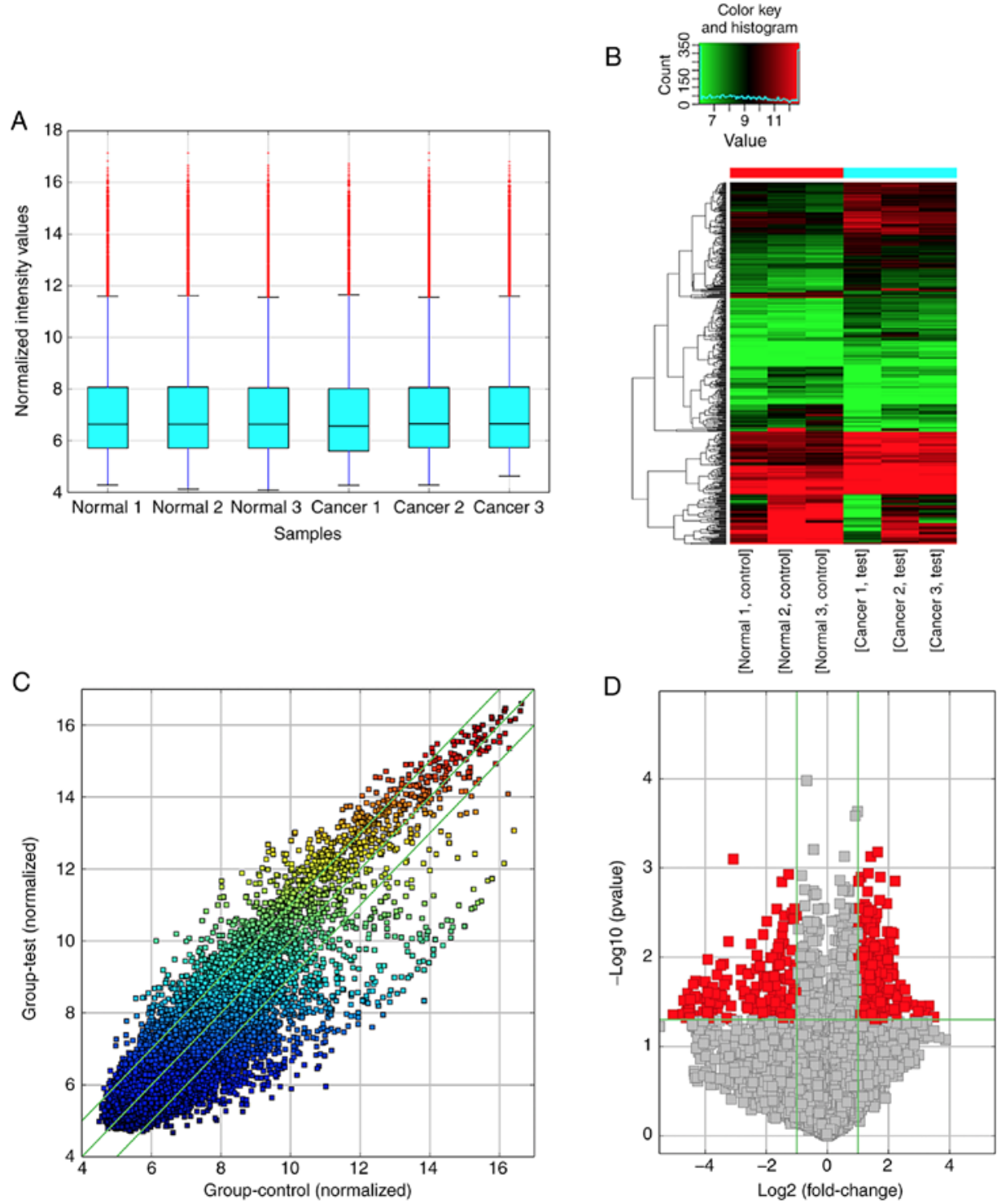

Figure 1. Overview of the microarray expression data. (A) The box plot was used to reveal the normalized intensities of the profile distributions of circRNAs (B) Hierarchical clustering of the differentially expressed circRNA expression data in BC tissues and control groups. The expression intensity was represented by different colors. (C) The scatter plot revealed the variation of circRNA expression between BC tissues and control groups. The values of the $\mathrm{x}$ - and $\mathrm{y}$-axes in the scatter plot were the normalized signal values of the samples (log2-scaled). The green fold-change lines represented 2-fold changes, thus circRNAs above or below the green lines indicated $>2.0$-fold upregulation or downregulation. (D) A volcano plot was applied to visualize differentially expressed circRNAs The vertical green lines indicated a 2.0 -fold $(\log 2$-scaled) up and down, respectively. The horizontal green line represents $\mathrm{P}=0.05$ (-log 10 -scaled). The red squares in the plot represent the differentially expressed circRNAs with statistical significance. BC, bladder cancer; ANNB tissues, adjacent non-neoplastic bladder tissues; circRNA, circular RNA.

The final score is the degree of staining multiplied by the intensity.

Statistical analysis. Quantitative data are presented as the mean \pm SEM. One-way ANOVA analysis and paired Student's t-test were used to assess significance among groups. Following one-way ANOVA, Tukey's post hoc test was performed. SPSS software (v19.0; SPSS, Inc.) was used to process all statistical analyses. A receiver operating characteristic (ROC) curve was generated to evaluate its diagnostic value and following calculation of a binomial exact-confidence interval to determine the area under the ROC curve (AUC). The associations between hsa_circRNA_100876 expression and prognosis of BC patients were evaluated using Kaplan-Meier survival analysis and log-rank tests. All tests were performed in triplicate. A
P-value $<0.05$ was considered to indicate a statistically significant difference.

\section{Results}

Overview of circRNA expression in paired bladder tissues. We selected three pairs of primary BC and ANNB tissues with similar clinicopathologic features to perform circRNA microarray for screening dysregulated circRNAs in BC. With a threshold of $\mathrm{FC} \geq 2.0$ and $\mathrm{P}<0.05,512$ differentially expressed circRNAs were identified following scanning and normalization, with 340 circRNAs significantly upregulated and 172 significantly downregulated. As revealed in Table SII, the top 10 regulated circRNAs were presented, along with other detailed information on P-value, FDR, strand, chromosome, 

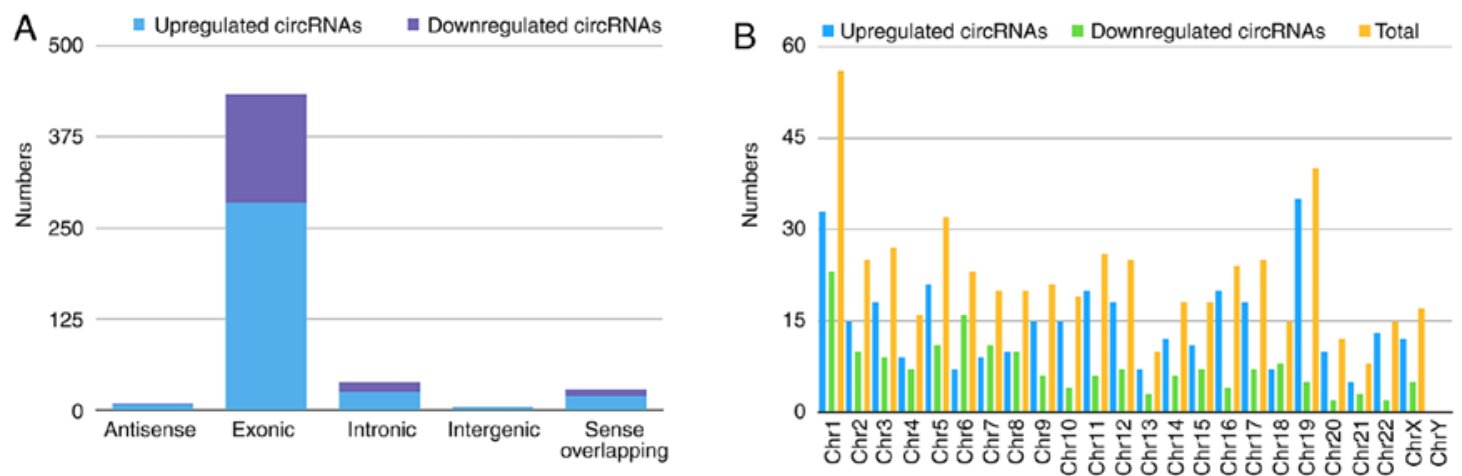

Figure 2. Distribution and classification of circRNAs. (A) Classification of the differentially expressed circRNAs is presented in the bar diagram. (B) The number of differentially expressed circRNAs detected in each chromosome. circRNAs, circular RNAs.
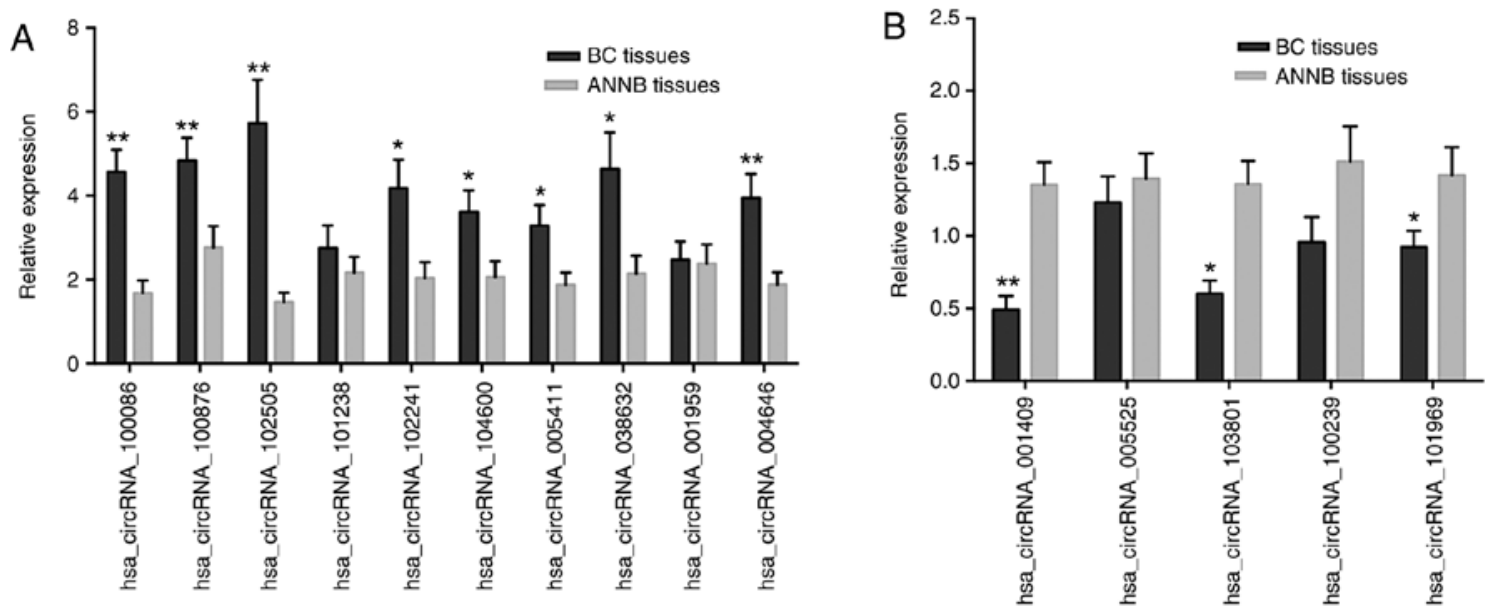

Figure 3. RT-qPCR validation of differentially expressed circRNAs. (A) Ten randomly selected circRNAs were validated to be upregulated in BC tissues compared with ANNB tissues by RT-qPCR. (B) Five randomly selected circRNAs were validated to be downregulated in BC tissues compared with ANNB tissues by RT-qPCR. Data were expressed as the mean \pm SEM. ${ }^{*} \mathrm{P}<0.05$ and ${ }^{* *} \mathrm{P}<0.01$. BC, bladder cancer; ANNB tissues, adjacent non-neoplastic bladder tissues; circRNAs, circular RNAs; RT-qPCR, reverse transcription-quantitative PCR; SEM, standard error of mean.

circRNA type and gene symbols. The box plot indicated that the circRNA distribution among the six samples was nearly identical after normalization (Fig. 1A), and hierarchical clustering revealed clear variations in the expression profiles between BC and ANNB tissues (Fig. 1B). Differentially expressed circRNAs among samples were assessed by scatter-plot visualization (Fig. 1C) which revealed the variation of circRNA expression between BC tissues and control groups, and volcano map analysis (Fig. 1D) which was applied to visualize differentially expressed circRNAs.

Classification of the dysregulated circRNAs is presented in the bar diagram (Fig. 2A). The results identified 340 upregulated circRNAs comprising 285 exonic, 25 intronic, 19 sense-overlapping, eight antisense, and three intergenic regions, whereas the 172 downregulated circRNAs contained 148 exonic, 13 intronic, nine sense-overlapping, one intergenic, and one antisense in BC-tissue samples. Chromosomal distribution analysis indicated that most were located on chromosome 1, while few located on chromosome 21 and the $\mathrm{Y}$ chromosome (Fig. 2B).

$R T$-qPCR validation of selected identified dysregulated circRNAs. Fifteen circRNAs (ten upregulated and five downregulated) were randomly selected from the microarray for further verification by RT-qPCR analysis from 40 sets of BC and ANNB tissues. Eleven of the 15 circRNAs were verified as significantly differentially expressed in cancer tissues, with eight upregulated and three downregulated (Fig. 3A and B). The results revealed upregulation of hsa_circRNA_100086, _038632,_100876,_102241,_004646,_102505,_104600, and _005411 expression in BC tissues relative to their levels in ANNB tissues, whereas hsa_circRNA_001409,_101969, and _103801 were downregulated. These findings were consistent with microarray results.

$G O$ and KEGG pathway analyses of the differentially expressed circRNAs. To investigate how circRNAs regulate the expression of a target gene, nine circRNAs (7 of the nine circRNAs were upregulated and 2 were downregulated) were randomly selected from the validated circRNAs for GO and KEGG pathway analyses. GO analysis annotated genes targeted by the nine differentially expressed circRNAs in domains of molecular functions, biological processes and cellular components. KEGG pathway analysis indicated that several pathways involved in cancer were related to the dysregulated circRNAs. The results were ranked by fold enrichment [(Count/Pop.Hits)/ 
A

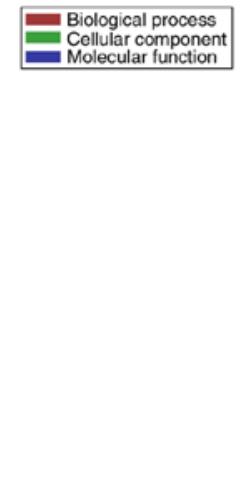

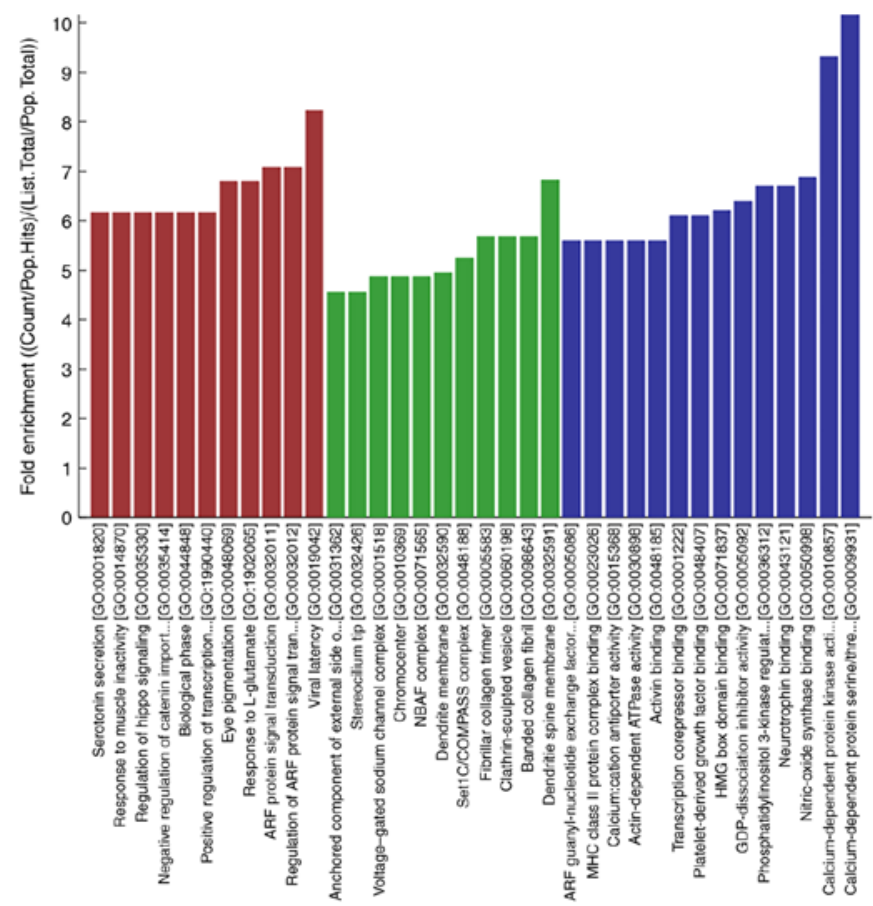

Biological process
Cellular component
Molecular function
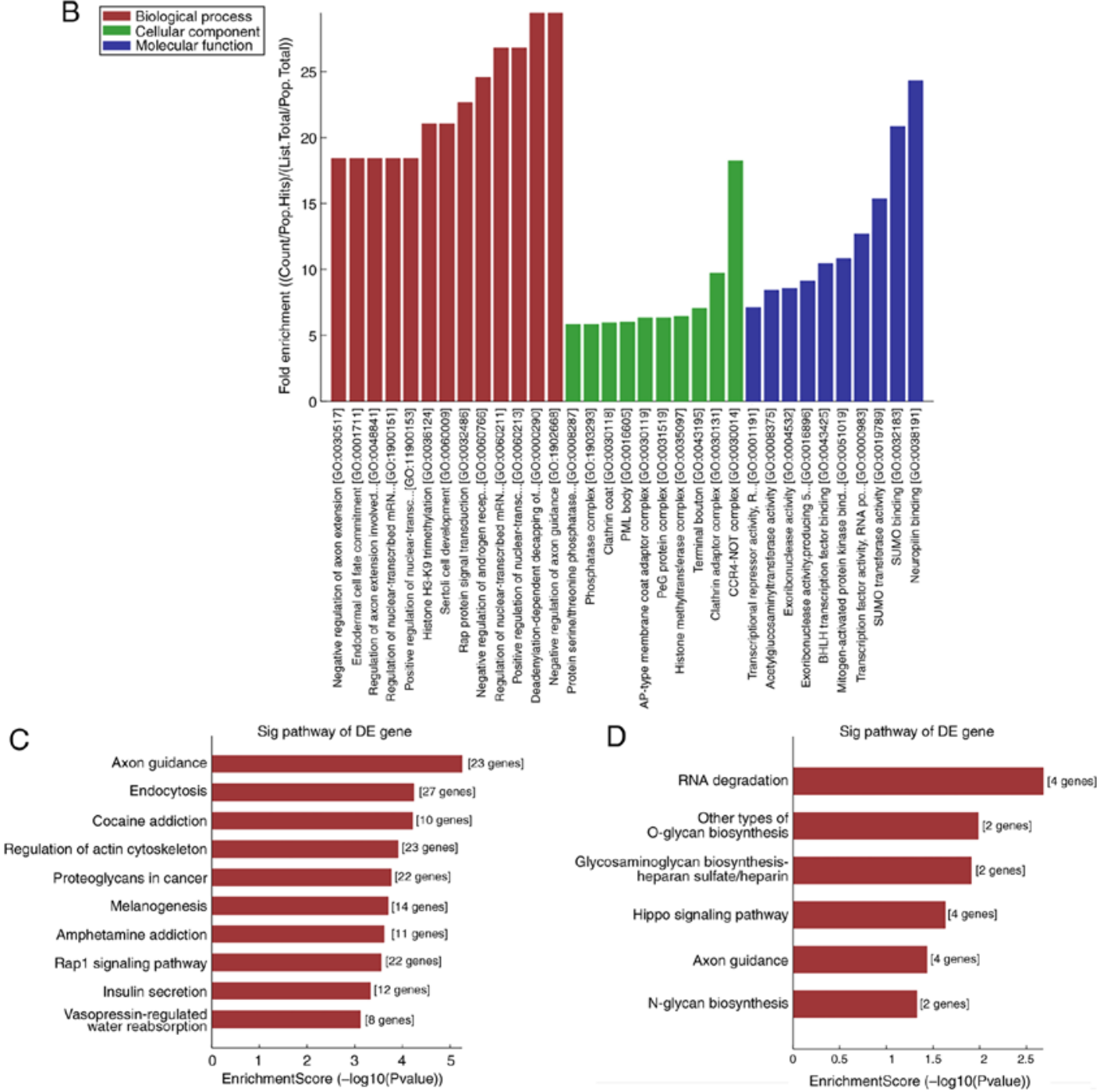

Figure 4. Top-ranked biological functions of hsa_circRNA_100876 and 038632 in GO analysis and Pathway analysis. (A) The top 10-fold enrichment scores in GO enrichment analysis on target genes of hsa_circRNA_038632. (B) The top 10-fold enrichment scores in GO enrichment analysis on target genes of hsa_circRNA_100876. Red bars represent BP, green bars represent CC and blue bars represent MF. (C) The top 10 enrichment scores in the Pathway analysis on target genes of hsa_circRNA_038632 are presented. (D) The top 6 enrichment scores in the Pathway analysis on target genes of hsa_circRNA_100876 are presented. circRNA, circular RNA; GO, Gene Ontology; BP, biological processes; CC, cellular components; MF, molecular functions. 
A

mRNA
mirnA
circRNA



B

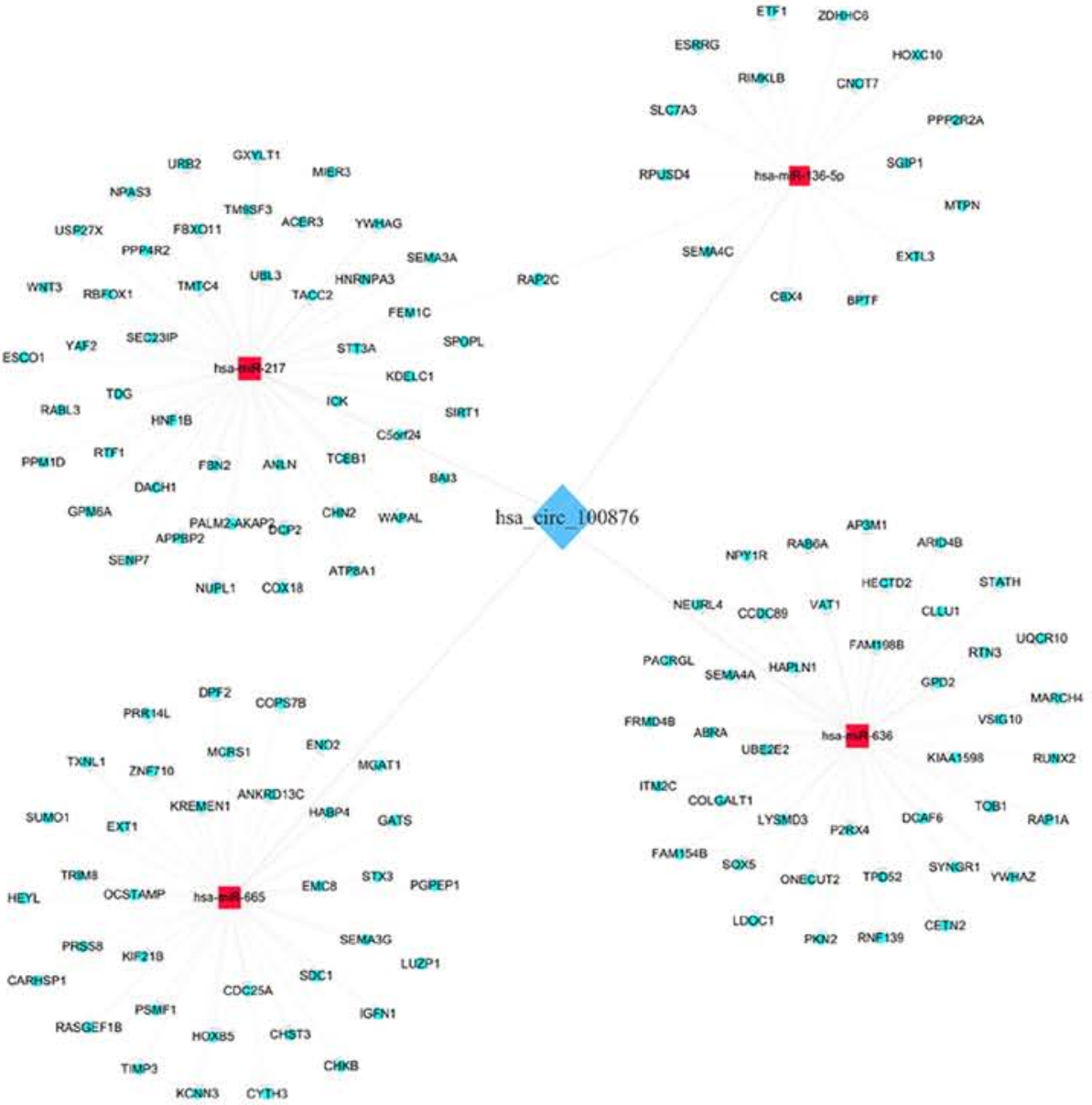

Figure 5. Construction of the circRNA-miRNA-mRNA regulation network. The visualization of (A) the circRNA-miRNA-mRNA regulation network for six candidate circRNAs and (B) the has_circRNA_100876-miRNA-mRNA interactions were predicted based on TargetScan and miRanda software. circRNA, circular RNA; miRNA, microRNA. 

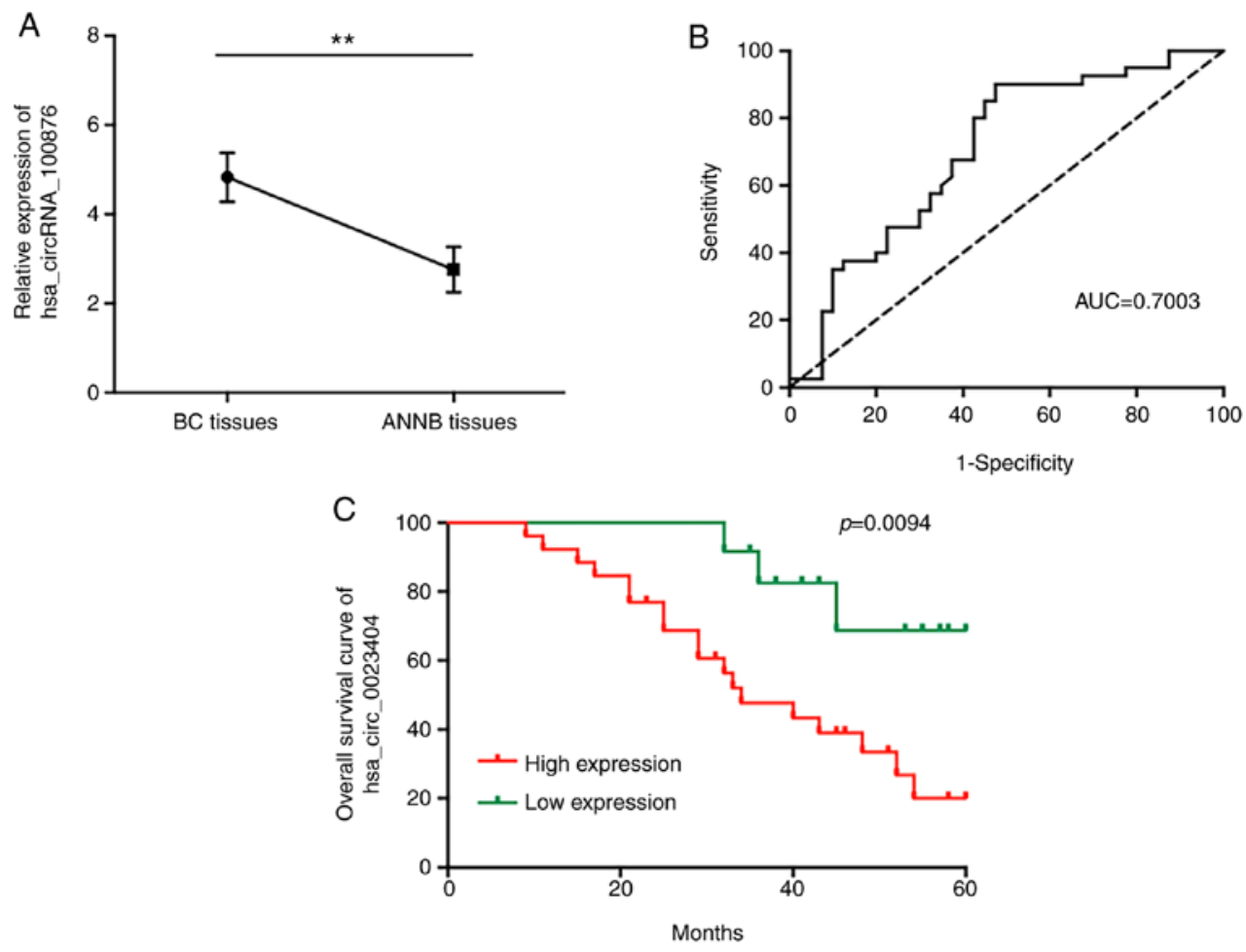

Figure 6. Relative expression of hsa_circRNA_100876 and its clinical significance in BC tissues. (A) Hsa_circRNA_100876 expression was validated in 40 pairs of BC tissues and ANNB tissues by RT-qPCR. (B) The ROC curve was used to evaluate the potential diagnostic value of hsa_circRNA_100876; the AUC was 0.7003. (C) Kaplan-Meier overall survival curve based on the expression level of hsa_circRNA_100876. Patients with high expression exhibited reduced survival time. BC, bladder cancer; ANNB tissues, adjacent non-neoplastic bladder tissues; circRNA, circular RNA; RT-qPCR, reverse transcription-quantitative PCR; ROC, receiver operating characteristic curve; AUC, area under the ROC curve. ${ }^{* *} \mathrm{P}<0.01$.

(List.Total/ Pop.Total)] and revealed enrichment of multiple critical biological functions associated with $\mathrm{BC}$ progression (results for hsa_circRNA_100876 and_038632 are presented in Fig. 4A-D). In addition, the supplementary results of GO and pathway analyses are presented in the Figs. S1-S7, identifying multiple items associated with cancer progression. For example, hsa_circRNA_102241 was related to 51 pathways, of which the top 1 pathway was related with 'proteoglycans in cancer'. As is known, some proteoglycans have pro- and anti-angiogenic activities, whereas other proteoglycans can also directly affect cancer growth by modulating key signaling pathways.

Construction an interaction network of ceRNA. To visualize a circRNA-regulatory network, interactions between the six confirmed differentially expressed circRNAs and their association with miRNAs and target genes were predicted, followed by construction of a unified interaction-network model. The co-expression pattern of circRNA-miRNA-mRNA is presented in Fig. 5A. These results indicated potential roles for the identified circRNAs as endogenous RNAs capable of altering target gene expression. In addition, a ceRNA network of hsa_circRNA_100876-miRNA-mRNA is presented in Fig. 5B. A total of 4 miRNAs (miR-136-5p, miR-217, miR-665 and miR-636) and corresponding target mRNAs were predicted to have an interaction with hsa_circRNA_100876 in the present study. For each of the four identified interacting miRNAs, the intersection of predictions from TargetScan and miRanda were used to find their targeting protein coding genes. A total of 140 miRNA-regulating protein coding genes were identified for hsa_circRNA_100876. It was revealed that hsa circRNA_100876 may be highly correlated and co-expressed with mRNAs. This provides us with another research strategy to explore the mechanism of hsa_circRNA_100876 by identifying its associated mRNAs and investigating whether it can play a role by regulating the expression of certain associated mRNAs.

Clinicopathologic assessment of hsa_circRNA_100876 expression in $B C$ patients. To determine possible roles of the dysregulated circRNAs in BC carcinogenesis, hsa_circRNA_100876 was selected for further study. The results revealed that hsa_circRNA_100876 was located on chromosome 11 (71668272-71671937) and differentially overexpressed in BC tissues relative to ANNB tissues (Fig. 6A). ROC curve analysis revealed an AUC value of 0.7003 (Fig. 6B), with a specificity of $52.5 \%$ and sensitivity of $90 \%$, suggesting the potential diagnostic efficacy of hsa_circRNA_100876 as a $\mathrm{BC}$ biomarker.

To confirm the clinical value of these molecular differences, analyses were performed to evaluate associations between hsa_circRNA_100876 expression and clinicopathologic features. As revealed in Table I, increased levels of hsa circRNA_100876 were significantly associated with T stage and lymphatic metastasis in $\mathrm{BC}$ patients $(\mathrm{P}<0.05)$, although the expression level was not related to sex, age, histological grade, or recurrence. Furthermore, univariate analysis indicated that the hsa_circRNA_100876 expression, T stage, and lymphatic metastasis were significantly related to the overall survival (OS) of patients $(\mathrm{P}<0.05)$, and multivariate analysis revealed 
Table I. Relationship between the expression levels of hsa_circRNA_100876 and clinicopathological features in bladder cancer patients.

\begin{tabular}{|c|c|c|c|c|c|}
\hline Features & Group & Case & High expression & Low expression & P-value \\
\hline \multirow[t]{2}{*}{ Age (years) } & $\leq 60$ & 14 & 8 & 6 & 0.336 \\
\hline & $>60$ & 26 & 18 & 8 & \\
\hline \multirow[t]{2}{*}{ Sex } & Male & 29 & 20 & 9 & 0.311 \\
\hline & Female & 11 & 6 & 5 & \\
\hline \multirow[t]{2}{*}{ Histological grade } & Low grade & 13 & 8 & 5 & 0.509 \\
\hline & High grade & 27 & 18 & 9 & \\
\hline \multirow[t]{2}{*}{ T Stage } & $\mathrm{T} 1-\mathrm{T} 2$ & 11 & 4 & 7 & $0.026^{\mathrm{a}}$ \\
\hline & $\mathrm{T} 3-\mathrm{T} 4$ & 29 & 22 & 7 & \\
\hline \multirow[t]{2}{*}{ Recurrence } & Yes & 12 & 8 & 4 & 0.591 \\
\hline & No & 28 & 18 & 10 & \\
\hline \multirow[t]{2}{*}{ Lymphatic metastasis } & Yes & 15 & 13 & 2 & $0.027^{\mathrm{a}}$ \\
\hline & No & 25 & 13 & 12 & \\
\hline
\end{tabular}

${ }^{\mathrm{a}} \mathrm{P}<0.05$, high vs. low expression.

Table II. Univariate and multivariate analysis for overall survival.

\begin{tabular}{|c|c|c|c|c|c|c|}
\hline \multirow[b]{2}{*}{ Parameters } & \multicolumn{3}{|c|}{ Univariate } & \multicolumn{3}{|c|}{ Multivariate } \\
\hline & HR & $95 \% \mathrm{CI}$ & P-value & HR & $95 \% \mathrm{CI}$ & P-value \\
\hline T stage & 2.730 & $1.173-6.524$ & $0.029^{\mathrm{a}}$ & 3.218 & $1.076-9.625$ & $0.037^{\mathrm{a}}$ \\
\hline Lymphatic metastasis & 2.291 & $1.023-6.820$ & $0.048^{\mathrm{a}}$ & 2.231 & $0.921-5.406$ & 0.076 \\
\hline Hsa_circRNA_100876 expression & 3.193 & $1.329-7.669$ & $0.009^{\mathrm{b}}$ & 3.704 & $1.076-12.753$ & $0.038^{\mathrm{a}}$ \\
\hline
\end{tabular}

${ }^{\mathrm{a}} \mathrm{P}<0.05$ and ${ }^{\mathrm{b}} \mathrm{P}<0.01$.

hsa_circRNA_100876 expression and lymphatic metastasis as independent factors affecting BC patient prognosis (Table II). Moreover, Kaplan-Meier survival analysis used to estimate the association between the hsa_circRNA_100876 patient prognosis post-surgery indicated that increased hsa circRNA_100876 expression was correlated with markedly shorter OS duration (Fig. 6C).

Hsa_circRNA_100876 inhibition suppresses BC cell proliferation. To confirm the role of hsa_circRNA_100876 in cell proliferation, specific siRNA targeting hsa_circRNA_100876 were transfected into BC cells (EJ and T24), resulting in a significant decrease in hsa_circRNA_100876 expression (Fig. 7A and $\mathrm{B} ; \mathrm{P}<0.05$ ). The results of $\mathrm{CCK}-8$ assays revealed that, compared to the si-NC group, the proliferation abilities of EJ and T24 cells transfected with si-hsa_circRNA_100876 were significantly reduced (Fig. $7 \mathrm{C}$ and $\mathrm{D} ; \mathrm{P}<0.05$ ), revealing that knockdown of hsa_circRNA_100876 suppressed proliferation of $\mathrm{BC}$ cells.

Overexpression of hsa_circRNA_100876 downregulates miR-136-5p and upregulates $C B X \overline{4}$ levels. We constructed a network of circRNA-miRNA-mRNA interactions to visualize potential relationships based on circRNA microarray data. Prediction of miRNA targets revealed that miR-136-5p shared binding sites with hsa_circRNA_100876 (Fig. 8A), and the binding sites were further validated by luciferase reporter assay (Fig. 8C). Furthermore, the same complementary binding sites were revealed between miR-136-5p and CBX4 (Fig. 8A), which was further validated through luciferase reporter assay (Fig. 8E). Moreover, RT-qPCR analysis confirmed significant downregulation of miR-136-5p (Fig. 8B) in BC tissues relative to ANNB tissues, along with its negative correlation with hsa_circRNA_100876 expression $(\mathrm{r}=-0.7842, \mathrm{P}<0.001)$ (Fig. 8F). Furthermore, it was observed that CBX4 levels were aberrantly upregulated in BC tissues (Fig. 8D), revealing a possible positive correlation with hsa_circRNA_100876 expression and a negative correlation with miR-136-5p expression $(r=0.7619$ and $-0.6404, \mathrm{P}<0.001$, respectively) (Fig. $8 \mathrm{G}$ and $\mathrm{H}$ ). Western blot analysis indicated increased $\mathrm{CBX} 4$ levels in BC tissues as compared with ANNB tissues, which was consistent with the RNA levels (Fig. 9A). Additionally, immunohistochemical staining results confirmed the increased CBX4 levels in $\mathrm{BC}$ tissues and relatively low levels in ANNB tissues (Fig. 9B). 

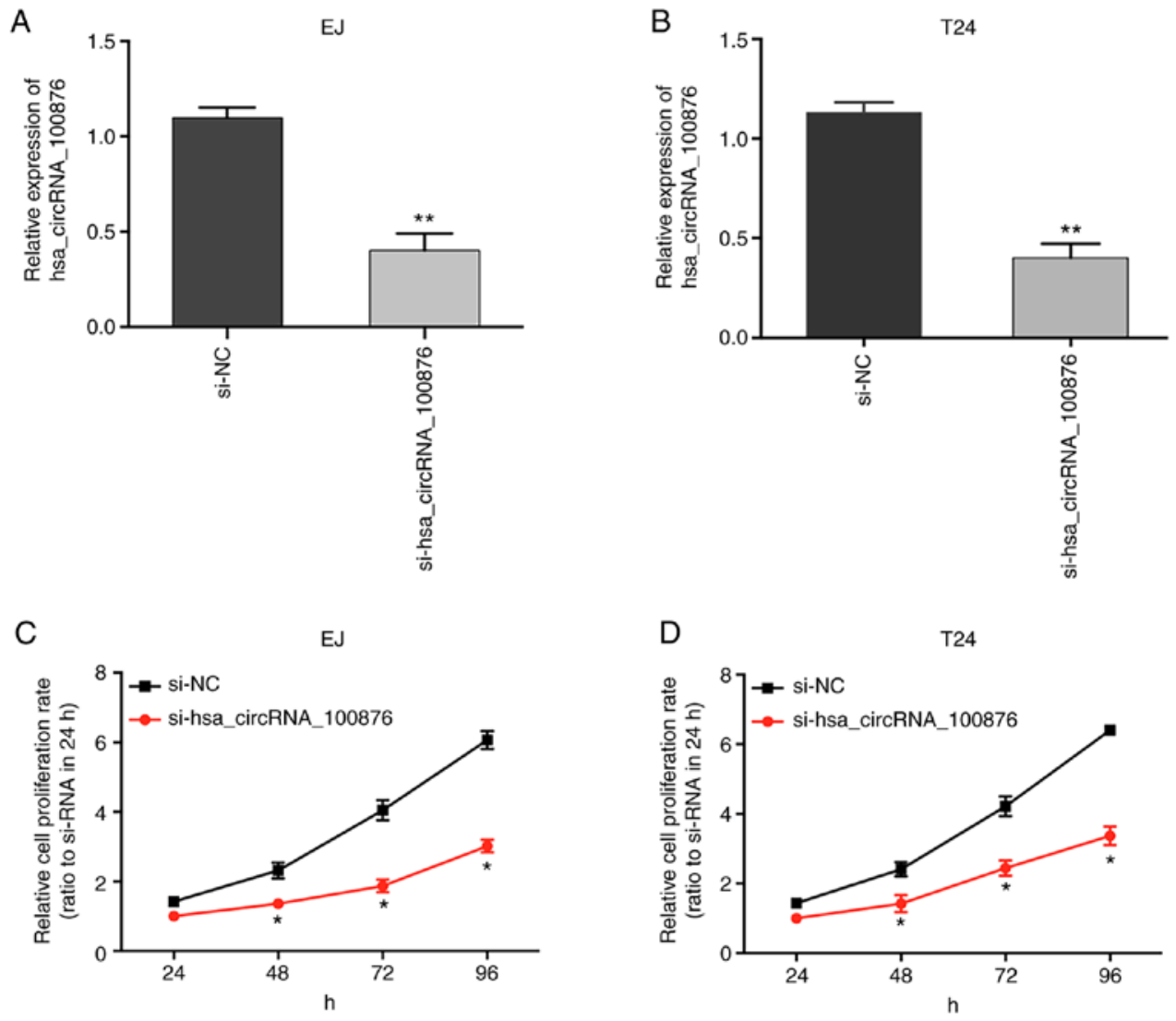

Figure 7. Knockdown of hsa_circRNA_100876 suppresses BC cell proliferation in vitro. (A and B) Expression of hsa_circRNA_100876 in EJ and T24 cell lines transfected with specific siRNAs targeting hsa_circRNA_100876. (C and D) CCK-8 assays revealed the proliferation in the hsa_circRNA_100876-knockdown group and the control group. Data were expressed as the mean \pm SEM. ${ }^{*} \mathrm{P}<0.05$ represents a statistically significant difference. ${ }^{* *} \mathrm{P}<0.01$. circRNA, circular RNA; BC, bladder cancer; si-, small interfering; NC, negative control.

\section{Discussion}

The molecular mechanisms associated with BC progression continue to be the focus of laboratory and clinical investigations, with recent findings identifying candidate miRNAs and lncRNAs involved in BC (24-26). As a new member of ncRNAs, circRNAs have attracted much attention for their involvement with disease and miRNA regulation $(27,28)$. CircRNAs are abundant, conserved, stable, and cell-type specific molecules involved in regulating cell function (29). Roles for circRNAs in diseases, such as diabetes (30) and those affecting the nervous (31) and cardiovascular systems (32), and tumorigenesis have received increased attention. For instance, a recent study has reported that hsa-circ-0000190 may be considered as a potential diagnostic biomarker for gastric cancer according to its downregulation in cancer tissues (33). Circ-ITCH was reported to be overexpressed in patients with lung cancer (34), and hsa_circRNA_100876 was associated with lymph node metastasis and stage of lung cancer (35). Furthermore, six circRNAs were identified to play important roles in carcinogenesis as ceRNA for the regulation of the miRNA-mRNA network in colorectal cancer (36). These findings indicate the key roles of circRNAs as biomarkers in early diagnosis and prognosis of cancers, as well as potential therapeutic targets. Furthermore, circRNAs have been identified in urologic neoplasms (37); however, their roles in BC remain largely unknown.
To investigate the possible effects of circRNAs on BC, 512 differentially expressed circRNAs from paired BC and ANNB tissues were identified. RT-qPCR analysis of the dysregulated circRNAs confirmed that 11 of the results were statistically significant $(\mathrm{P}<0.05)$ and consistent with microarray results. Reasons for the verification inconformity results compared with the chip are: Firstly, the microarray chip technology itself has a certain false positive rate, and the high cost of screening chips leads to a limited number of cases for screening of circRNAs. Additionally, different results may occur due to the inherent differences among patients, such as the degree of pathological differentiation and cancer stage and grade. Moreover, differences in the standardization process between the microarray and RT-qPCR could also lead to variations in the results.

The potential function of the differentially expressed circRNAs was elucidated by GO and KEGG analyses. The results indicated their relationship with the aberrant expression of several mRNAs, as well as biological pathways alterations, suggesting their potential roles in BC.GO analysis indicated that hsa_circRNA_038632 was associated with ADP-ribosylation factors (ARFs), which was associated with p53 degradation and downregulation of p53 transcription. Certain oncogenes and regulatory factors can regulate p53 function through ARFs, leading to several cancers (38-41). Additionally, studies have reported dysregulated ARF levels in $\mathrm{BC}$ along with abnormal promoter methylation involved in BC development, suggesting 
A hsa_circRNA_100876 $\rightarrow 5^{\prime}$-tctcTCTCCAGAAGAGCAATGGAGg- $3^{\prime}$

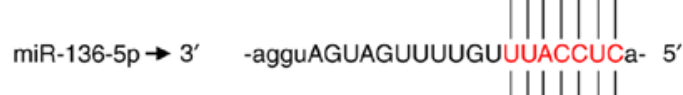

$\mathrm{CBX} 4 \rightarrow 5^{\prime} \quad$-UGAAUGUAAAUAUUAAAUGGAGA- $3^{\prime}$
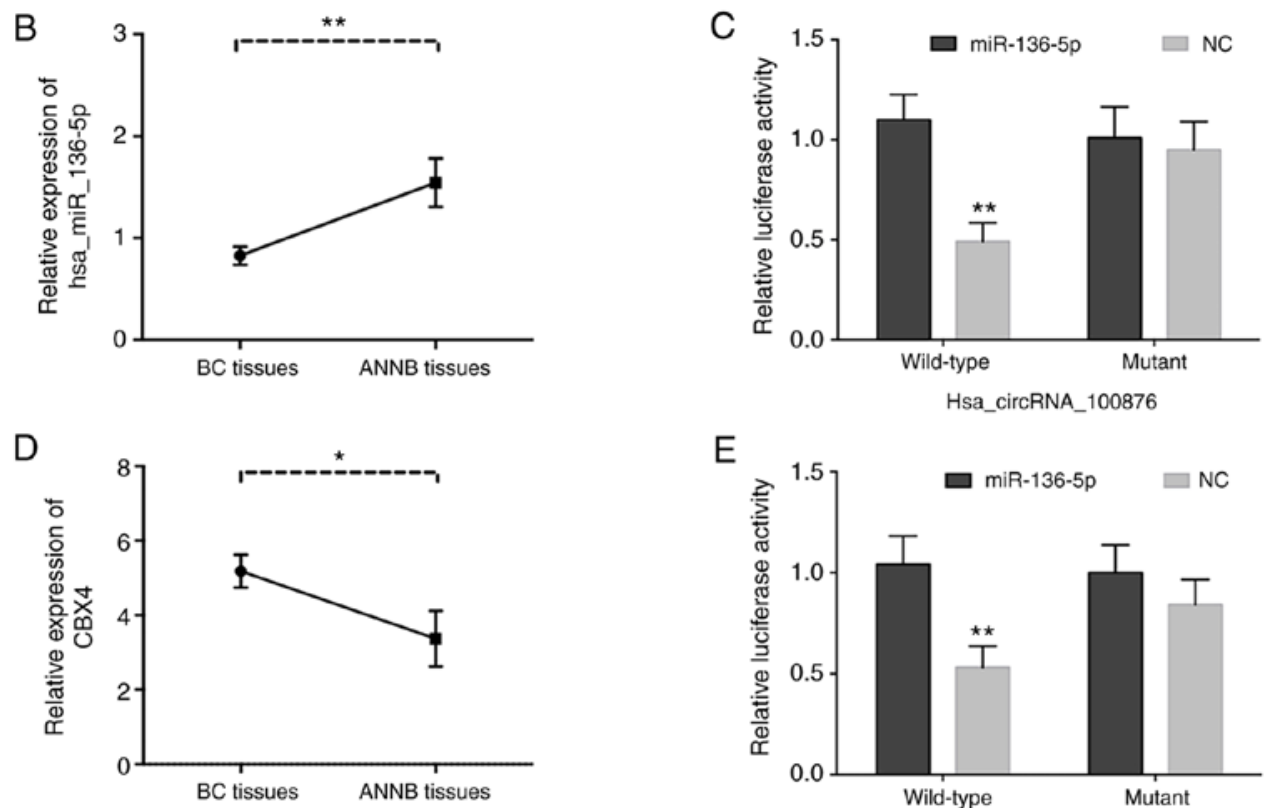

E
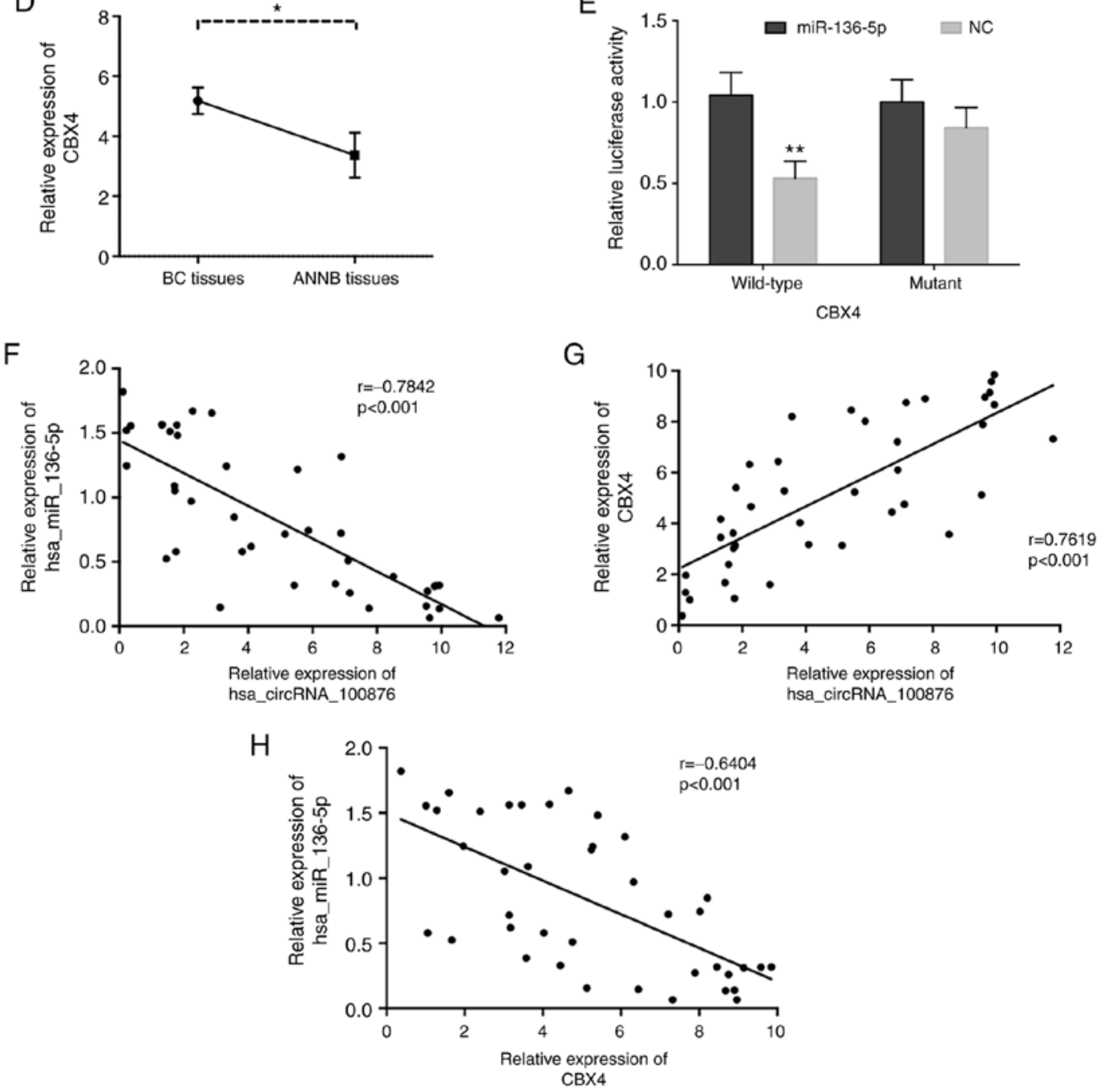

Figure 8. Correlations of hsa_circRNA_100876, miR-136-5p and CBX4. (A) The identical putative binding sites of miR-136-5p on the hsa_circRNA_100876 and CBX4. (B) The relative expression of miR-136-5p was validated in 40 pairs of BC tissues and ANNB tissues by RT-qPCR. (C) A luciferase reporter assay validated the binding of has_circRNA_100876 and miR-136-5p. (D) The relative expression of CBX4 was validated in 40 pairs of BC tissues and ANNB tissues by RT-qPCR. (E) A luciferase reporter assay validated the binding of miR-136-5p and CBX4. (F) Hsa_circRNA_100876 expression was negatively correlated with miR-136-5p expression in BC tissues $(\mathrm{r}=-0.7842, \mathrm{P}<0.001)$. (G) Hsa_circRNA_100876 expression was positively correlated with CBX4 expression in $\mathrm{BC}$ tissues $(\mathrm{r}=0.7619, \mathrm{P}<0.001)$. (H) CBX4 expression was negatively correlated with miR-136-5p expression in $\mathrm{BC}$ tissues $(\mathrm{r}=-0.6404, \mathrm{P}<0.001)$. CBX4, chromobox 4; BC, bladder cancer; ANNB tissues, adjacent non-neoplastic bladder tissues; RT-qPCR, reverse transcription-quantitative PCR; NC, negative control. ${ }^{*} \mathrm{P}<0.05,{ }^{* * *} \mathrm{P}<0.01$.

it as a potential prognostic marker of BC $(42,43)$. Moreover, GO analysis revealed that an hsa_circRNA_100876 target gene was involved in Ras-related protein (RAP) signal transduction and the $\mathrm{CC}$ chemokine receptor 4-negative regulator 

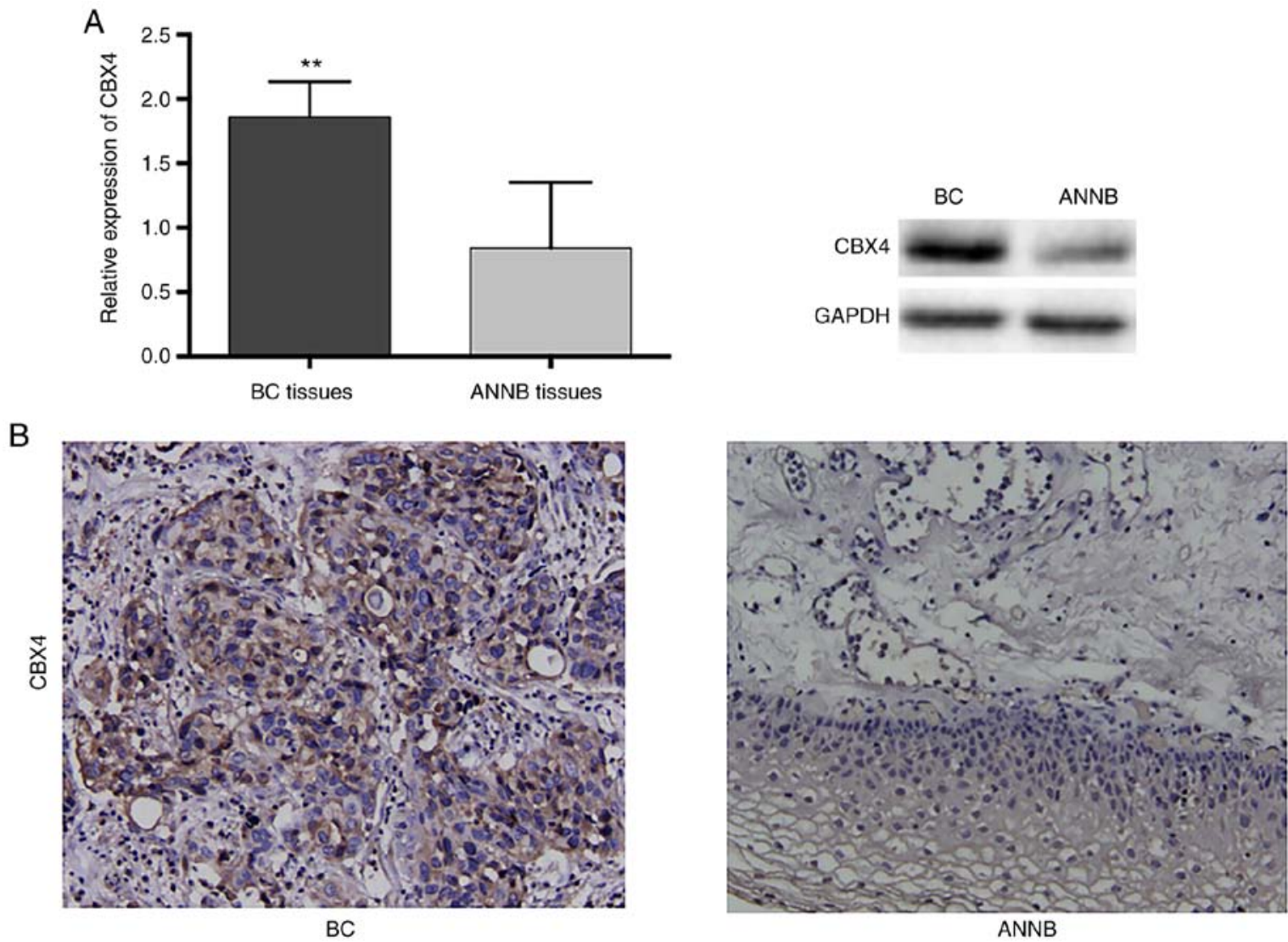

Figure 9. Western blotting and immunohistochemical staining of CBX4 expression on BC tissues. (A) Western blotting revealed that $\mathrm{BC}$ tissues had higher CBX4 expression levels in contrast with ANNB tissues. (B) High expression of CBX4 could be observed in BC tissues compared to ANNB tissues. CBX4 staining was found in the nuclei and partly in the cytoplasm of normal and tumor tissue. ${ }^{* *} \mathrm{P}<0.01$. CBX4, chromobox 4 ; $\mathrm{BC}$, bladder cancer; ANNB tissues, adjacent non-neoplastic bladder tissues.

of transcription (CCR4-NOT) complex. RAP plays an important part in the mitogen-activated protein kinase (MAPK) pathway, which acts as a central role in tumorigenesis by promoting cancer development and progression, including that of BC (44). Additionally, the CCR4-NOT complex plays extensive roles in gene regulation, such as transcription regulation, mRNA attenuation and quality control, translation inhibition, and protein ubiquitination (45). Therefore, these differentially expressed circRNAs may exert their biological functions by affecting the expression of target genes associated with pathways involved in promoting BC development. KEGG analysis identified multiple pathways associated with cancer progression. The results revealed that hsa_circRNA_038632 was related to 61 pathways, 28 of which are associated with cancers, including classic cancer pathways involving p53, Wnt, and Ras signaling pathway. Among the identified pathways related to malignancy, we discovered a BC-specific pathway, with the corresponding mRNAs identified as playing key roles in tumor aggressiveness $(46,47)$. Moreover, six pathways related to hsa_circRNA_100876 included four malignancy-related pathways and the Hippo-signaling pathway was recently confirmed as being involved in BC development (48). These findings suggested that the identified circRNAs may affect the expression of target genes associated with different BC-related pathways directly or indirectly.

A previous study reported that circRNAs are enriched with miRNA response elements (MREs), which could be regarded as miRNA sponges to adsorb miRNA and eliminate their inhibitory effects on target mRNA, thereby upregulating protein translation as an important aspect of posttranscriptional regulation (13). The mechanisms associated with circRNA functions as miRNA sponges related to $\mathrm{BC}$ have been reported in several studies. Zhong et al (49) reported that circRNA MYLK reduced miR-29a activity through sponge adsorption to advance the progression of $\mathrm{BC}$ by measuring the vascular endothelial growth factor (VEGF)/VEGF receptor-2 signaling pathway. Different from traditional linear miRNA sponges with only one MRE, circRNA contain several MREs. In a previous study, Li et al (50) reported that circ-ITCH competitively adsorbed miR-17, miR-214 and miR-7, leading to an increased expression of circ-ITCH and degraded phosphorylated Dvl2, thereby hindering esophageal cancer tumor growth. Given that a single circRNA could regulate numerous downstream genes via a common miRNA target, our results suggested that these circRNAs may potentially act as drivers of cancer through highly enriched signaling pathways. CircRNAs may represent novel biomarkers for tumor diagnosis and prognosis, as well as miRNA inhibitors involved in antitumor therapy.

Identification of miRNA-binding sites via circRNA microarray analysis allows investigation of their indirect effects on several functional mRNAs, resulting in possible identification of circRNA function(s). The microarray analysis identified five miRNA-binding sites in circRNAs, and we anticipate that the possible BC-specific biological function and mechanism of 
circRNAs could be identified through these miRNAs or target mRNAs that share the same MREs with these miRNAs. It was observed that as a tumor suppressor, miRNA-136-5p played an important role in tumor progression related to kidney (51), lung (52), and breast cancer (53). A recent study concerning miRNA screening for BC revealed that miRNA-136-5p was among the top 10 downregulated miRNAs, but without large sample validation (54). In the present study, RT-qPCR analysis of 40 paired tissue samples confirmed the downregulation of miR-136-5p in $\mathrm{BC}$, with the findings consistent with the microarray results. Bioinformatics analysis subsequently identified miR-136-5p-specific MREs present in both hsa_circRNA 100876 and mRNA-CBX4, which was validated by luciferase assay. Therefore, it was surmised that there may be important regulatory relationships between hsa_circRNA_100876, miR-136-5p, and CBX4 resulting in alteration of BC-related activity.

This study, to the best of our knowledge, is the first study reporting significant upregulation of hsa_circRNA_100876 levels in BC tissues. Additionally, it revealed that upregulated expression of hsa_circRNA_100876 was positively correlated with lymph node metastasis, $\mathrm{T}$ stage and significantly shorter OS, suggesting that hsa_circRNA_100876 may play a critical role in biological functions associated with the occurrence and development of BC.

CBX proteins are members of the PcG family of proteins, which participate in the malignant progression of numerous human cancers. CBX4 is a unique protein with enzymatic activity in the CBX family, and can act as small ubiquitin-like modifier (SUMO) E3 ligase in SUMO modification, which plays a significant role in regulation of transcription, DNA repair structure, and alteration of chromatin (55). Abnormal SUMO modifications are related to numerous diseases, including cancers (56). CBX4 regulates the levels of various proteins via SUMO E3 activity, and its expression in malignant tumors has become a research hotspot (57). Li et al (58) reported that CBX4 can upregulate hypoxia-induced VEGF expression via SUMO modification of two lysine sites in hypoxia-inducible-1a, thereby promoting angiogenesis in hepatocellular carcinoma. In breast cancer, it was demonstrated that CBX4 could reduce the cell proliferation induced by estrogen via inhibiting estrogen-signal transduction by SUMO modification of zinc finger protein 131 (59). In the present study, GO analysis revealed that hsa_circRNA_100876 was enriched in association with PcG complex, SUMO transfer activity, and SUMO binding, with the corresponding genes universally including CBX4. The results further confirmed the biological role of $\mathrm{CBX} 4$ and revealed a potential relationship between hsa_circRNA_100876 and CBX4. The subsequent correlation analysis revealed a negative correlation between hsa_circRNA_100876 and CBX4 expression and that of miR-136-5p. Additionally, hsa_circRNA_100876 and CBX4 expression was positively correlated. These results suggested that hsa_circRNA_100876 may act as sponge for miR-136-5p, resulting in increased in $\mathrm{CBX} 4$ levels, thereby altering $\mathrm{BC}$ progression.

Currently, the molecular mechanisms associated with circRNAs in BC remain unclear. The present study demonstrated that numerous circRNAs were dysregulated and these aberrantly expressed circRNAs may serve key functions to the occurrence and development of $\mathrm{BC}$. The results provided novel insight into the complex biological functions of circRNAs in the process of $\mathrm{BC}$ carcinogenesis and circRNAs could serve as potential biomarkers for BC. Furthermore, this is the first study reporting overexpression of hsa_circRNA_100876 in $\mathrm{BC}$ and the potential role of $\mathrm{CBX} 4$ in $\mathrm{BC}$. The relationship between hsa_circRNA_100876,miR-136-5p and CBX4 may be a crucial molecular mechanism related to BC. A future study will focus on elucidating the regulatory relationship between hsa_circRNA_100876 and CBX4 in association with BC.

\section{Acknowledgements}

The authors want to thank the Experimental Center of Shengjing Hospital (Shenyang, China) for technical support.

\section{Funding}

The present study was supported by grants from the Natural Science Foundation of Liaoning Province of China (grant no. 20170540988) and the Shenyang Science and Technology Program (grant no. 17-231-1-57).

\section{Availability of data and materials}

The datasets used and analyzed during the current study are available from the corresponding author on reasonable request.

\section{Authors' contributions}

SL contributed to the study design, data collection and interpretation, and writing of the manuscript. SL and YZ analyzed and interpreted the data and prepared the figures. XC and SL designed the study. YZ and $\mathrm{XC}$ contributed to the revision and editing of the manuscript and the submission of the manuscript for publication. All authors reviewed and approved the final manuscript

\section{Ethics approval and consent to participate}

The use of clinical data was approved by the Research Ethics Committee of Shengjing Hospital of China Medical University (Shenyang, China). Informed consent was provided by all subjects for inclusion in the study, and the research plan was approved by the Research Ethics Committee of Shengjing Hospital of China Medical University.

\section{Patient consent for publication}

Not applicable.

\section{Competing interests}

The authors declare that they have no competing interests.

\section{References}

1. Kaufman DS, Shipley WU and Feldman AS: Bladder cancer. Lancet 374: 239-249, 2009. 
2. Ferlay J, Soerjomataram I, Dikshit R, Eser S, Mathers C, Rebelo M, Parkin DM, Forman D and Bray F: Cancer incidence and mortality worldwide: Sources, methods and major patterns in GLOBOCAN 2012. Int J Cancer 136: E359-E386, 2015.

3. Siegel RL, Miller KD and Jemal A: Cancer statistics, 2018. CA Cancer J Clin 68: 7-30, 2018.

4. Kim WT, Kim YH, Jeong P, Seo SP, Kang HW, Kim YJ, Yun SJ, Lee SC, Moon SK, Choi Y, et al: Urinary cell-free nucleic acid IQGAP3: A new non-invasive diagnostic marker for bladder cancer. Oncotarget 9: 14354-14365, 2018.

5. Fatica A and Bozzoni I: Long non-coding RNAs: New players in cell differentiation and development. Nat Rev Genet 15: 7-21, 2014.

6. Li PF, Chen SC, Xia T, Jiang XM, Shao YF, Xiao BX and Guo JM: Non-coding RNAs and gastric cancer. World J Gastroenterol 20: 5411-5419, 2014

7. Qu S, Yang X, Li X, Wang J, Gao Y, Shang R, Sun W, Dou K and Li H: Circular RNA: A new star of noncoding RNAs. Cancer Lett 365: 141-148, 2015.

8. Greene J, Baird AM, Brady L, Lim M, Gray SG, McDermott R and Finn SP: Circular RNAs: Biogenesis, Function and Role in Human Diseases. Front Mol Biosci 4: 38, 2017.

9. Chen LL and Yang L: Regulation of circRNA biogenesis. RNA Biol 12: 381-388, 2015.

10. Zhang XO, Wang HB, Zhang Y, Lu X, Chen LL and Yang L: Complementary sequence-mediated exon circularization. Cell 159: 134-147, 2014

11. Cocquerelle C, Mascrez B, Hétuin D and Bailleul B: Mis-splicing yields circular RNA molecules. FASEB J 7: 155-160, 1993.

12. Salzman J, Chen RE, Olsen MN, Wang PL and Brown PO: Cell-type specific features of circular RNA expression. PLoS Genet 9: e1003777, 2013.

13. Vidal AF, Sandoval GT, Magalhaes L, Santos SE and Ribeiro-dos-Santos A: Circular RNAs as a new field in gene regulation and their implications in translational research. Epigenomics 8: 551-562, 2016.

14. Xu S, Zhou L, Ponnusamy M, Zhang L, Dong Y, Zhang Y, Wang Q, Liu J and Wang K: A comprehensive review of circRNA: From purification and identification to disease marker potential. PeerJ 6: e5503, 2018.

15. Zhang X, Zhou H, Jing W, Luo P, Qiu S, Liu X, Zhu M, Liang C, $\mathrm{Yu} \mathrm{M}$ and $\mathrm{Tu} \mathrm{J}$ : The circular RNA hsa circ 0001445 regulates the proliferation and migration of hepatocellular carcinoma and may serve as a diagnostic biomarker. Dis Markers 2018: 3073467, 2018.

16. Jiang MM, Mai ZT, Wan SZ, Chi YM, Zhang X, Sun BH and Di QG: Microarray profiles reveal that circular RNA hsa circ_0007385 functions as an oncogene in non-small cell lung cancer tumorigenesis. J Cancer Res Clin Oncol 144: 667-674, 2018.

17. Zhong Z, Lv M and Chen J: Screening differential circular RNA expression profiles reveals the regulatory role of circTCF25-miR-103a-3p/miR-107-CDK6 pathway in bladder carcinoma. Sci Rep 6: 30919, 2016.

18. Cai D, Liu Z and Kong G: Molecular and bioinformatics analyses identify 7 circular RNAs involved in regulation of oncogenic transformation and cell proliferation in human bladder cancer. Med Sci Monit 24: 1654-1661, 2018.

19. Enright AJ, John B, Gaul U, Tuschl T, Sander C and Marks DS MicroRNA targets in Drosophila. Genome Biol 5: R1, 2003.

20. Pasquinelli AE: MicroRNAs and their targets: Recognition, regulation and an emerging reciprocal relationship. Nat Rev Genet 13: 271-282, 2012.

21. Livak KJ and Schmittgen TD: Analysis of relative gene expression data using real-time quantitative PCR and the 2(-Delta Delta C(T)) method. Methods 25: 402-408, 2001.

22. Ashburner M, Ball CA, Blake JA, Botstein D, Butler $\mathrm{H}$, Cherry JM, Davis AP, Dolinski K, Dwight SS, Eppig JT, et al: Gene ontology: Tool for the unification of biology. Nat Genet 25 : 25-29, 2000.

23. Gerlich M and Neumann S: KEGG: Kyoto encyclopedia of genes and genomes. Nuclc Acids Res 28: 27-30, 2000.

24. Shi Z, Kadeer A, Wang M, Wen B, Li M, Huang J, Gao Y, Liu E, Liu D, Jia D and Liang C: The deregulation of miR-133b is associated with poor prognosis in bladder cancer. Pathol Res Pract 215: 354-357, 2019.

25. Yu QF, Liu P, Li ZY, Zhang CF, Chen SQ, Li ZH, Zhang GY and Li JC: MiR-103/107 induces tumorigenicity in bladder cancer cell by suppressing PTEN. Eur Rev Med Pharmacol Sci 22: 8616-8623, 2018.
26. Wang F, Zu Y, Zhu S, Yang Y, Huang W, Xie H and Li G: Long noncoding RNA MAGI2-AS3 regulates CCDC19 expression by sponging miR-15b-5p and suppresses bladder cancer progression. Biochem Biophys Res Commun 507: 231-235, 2018.

27. Li Y, Wan B, Liu L, Zhou L and Zeng Q: Circular RNA circMTO1 suppresses bladder cancer metastasis by sponging miR-221 and inhibiting epithelial-to-mesenchymal transition. Biochem Biophys Res Commun 508: 991-996, 2019.

28. Sun M,Zhao W, Chen Z,Li M,Li S, Wu B and Bu R:Circ_0058063 regulates CDK6 to promote bladder cancer progression by sponging miR-145-5p. J Cell Physiol 234: 4812-4824, 2019.

29. Zhao X, Wang Y, Yu Q, Yu P, Zheng Q, Yang X and Gao D: Circular RNAs in gastrointestinal cancer: Current knowledge, biomarkers and targeted therapy (Review). Int J Mol Med 46: 1611-1632, 2020.

30. Zhang JR and Sun HJ: Roles of circular RNAs in diabetic complications: From molecular mechanisms to therapeutic potential. Gene 763: 145066, 2020.

31. Hua L, Huang L, Zhang X, Feng H and Shen B: Knockdown of circular RNA CEP128 suppresses proliferation and improves cytotoxic efficacy of temozolomide in glioma cells by regulating miR-145-5p. Neuroreport 30: 1231-1238, 2019.

32. Gan J, Yuan J, Liu Y, Lu Z, Xue Y, Shi L and Zeng H: Circular RNA_101237 mediates anoxia/reoxygenation injury by targeting let-7a-5p/IGF2BP3 in cardiomyocytes. Int J Mol Med 45: 451-460, 2020.

33. Chen S, Li T, Zhao Q, Xiao B and Guo J: Using circular RNA hsa circ 0000190 as a new biomarker in the diagnosis of gastric cancer. Clin Chim Acta 466: 167-171, 2017.

34. Xiao-Long M, Kun-Peng Z and Chun-Lin Z: Circular RNA circ_HIPK3 is down-regulated and suppresses cell proliferation, migration and invasion in osteosarcoma. J Cancer 9: 1856-1862, 2018.

35. Yao JT, Zhao SH, Liu QP, Lv MQ, Zhou DX, Liao ZJ and Nan KJ: Over-expression of CircRNA_100876 in non-small cell lung cancer and its prognostic value. Pathol Res Pract 213: 453-456, 2017.

36. Yuan W, Peng S, Wang J, Wei C, Ye Z, Wang Y, Wang M, Xu H, Jiang S, Sun D, et al: Identification and characterization of circRNAs as competing endogenous RNAs for miRNA-mRNA in colorectal cancer. PeerJ 7: e7602, 2019.

37. Su Y, Du Z, Zhong G, Ya Y, Bi J, Shi J, Chen L, Dong W and Lin T: circ5912 suppresses cancer progression via inducing MET in bladder cancer. Aging (Albany NY) 11: 10826-10838, 2019.

38. Chaar I, Amara S, Elamine OE, Khiari M, Ounissi D, Khalfallah T, Ben Hmida A, Mzabi S and Bouraoui S: Biological significance of promoter hypermethylation of p14/ARF gene: Relationships to p53 mutational status in Tunisian population with colorectal carcinoma. Tumour Biol 35: 1439-1449, 2014.

39. Hsu HS, Wang YC, Tseng RC, Chang JW, Chen JT, Shih CM, Chen CY and Wang YC: 5' cytosine-phospho-guanine island methylation is responsible for p14ARF inactivation and inversely correlates with p53 overexpression in resected non-small cell lung cancer. Clin Cancer Res 10: 4734-4741, 2004.

40. Iida S, Akiyama Y, Nakajima T, Ichikawa W, Nihei Z, Sugihara K and Yuasa Y: Alterations and hypermethylation of the p14(ARF) gene in gastric cancer. Int J Cancer 87: 654-658, 2000.

41. Ito $T$, Nishida N, Fukuda $Y$, Nishimura $T$, Komeda $T$ and Nakao K: Alteration of the p14(ARF) gene and p53 status in human hepatocellular carcinomas. J Gastroenterol 39: 355-361, 2004.

42. Domínguez G, Carballido J, Silva J, Silva JM, García JM, Menéndez J, Provencio M, España P and Bonilla F: p14ARF promoter hypermethylation in plasma DNA as an indicator of disease recurrence in bladder cancer patients. Clin Cancer Res 8: 980-985, 2002

43. Berggren P, Kumar R, Sakano S, Hemminki L, Wada T, Steineck G, Adolfsson J, Larsson P, Norming U, Wijkström H and Hemminki K: Detecting homozygous deletions in the CDKN2A(p16(INK4a))/ARF(p14(ARF)) gene in urinary bladder cancer using real-time quantitative PCR. Clin Cancer Res 9: 235-242, 2003.

44. Liang Z, Xie W, Wu R, Geng H, Zhao L, Xie C, Li X, Zhu M, Zhu W, Zhu J, et al: Inhibition of tobacco smoke-induced bladder MAPK activation and epithelial-mesenchymal transition in mice by curcumin. Int J Clin Exp Pathol 8: 4503-4513, 2015.

45. Miller JE and Reese JC: Ccr4-Not complex: The control freak of eukaryotic cells. Crit Rev Biochem Mol Biol 47: 315-333, 2012. 
46. Noel N, Couteau J, Maillet G, Gobet F, D'Aloisio F, Minier C and Pfister C: TP53 and FGFR3 gene mutation assessment in urine: Pilot study for bladder cancer diagnosis. Anticancer Res 35: 4915-4921, 2015

47. Knowles MA: Role of FGFR3 in urothelial cell carcinoma: Biomarker and potential therapeutic target. World J Urol 25 581-593, 2007.

48. Dong L, Lin F, Wu W, Liu Y and Huang W: Verteporfin inhibits YAP-induced bladder cancer cell growth and invasion via Hippo signaling pathway. Int J Med Sci 15: 645-652, 2018.

49. Zhong Z, Huang M, Lv M, He Y, Duan C, Zhang L and Chen J: Circular RNA MYLK as a competing endogenous RNA promotes bladder cancer progression through modulating VEGFA/VEGFR2 signaling pathway. Cancer Lett 403: 305-317, 2017.

50. Li F, Zhang L, Li W, Deng J, Zheng J, An M, Lu J and Zhou Y: Circular RNA ITCH has inhibitory effect on ESCC by suppressing the Wnt/ $\beta$-catenin pathway. Oncotarget 6: 6001-6013, 2015.

51. Chen P, Zhao L, Pan X, Jin L, Lin C, Xu W, Xu J, Guan X, Wu X, Wang Y, et al: Tumor suppressor microRNA-136-5p regulates the cellular function of renal cell carcinoma. Oncol Lett 15: 5995-6002, 2018.

52. Shen S, Yue H, Li Y, Qin J, Li K, Liu Y and Wang J: Upregulation of miR-136 in human non-small cell lung cancer cells promotes Erk1/2 activation by targeting PPP2R 2A. Tumour Biol 35: 631-640, 2014.
53. Yan M, Li X, Tong D, Han C, Zhao R, He Y and Jin X: miR-136 suppresses tumor invasion and metastasis by targeting RASAL2 in triple-negative breast cancer. Oncol Rep 36: 65-71, 2016.

54. Scelfo A, Piunti A and Pasini D: The controversial role of the Polycomb group proteins in transcription and cancer: How much do we not understand Polycomb proteins? FEBS J 282: 1703-1722, 2015 .

55. Haindl M, Harasim T, Eick D and Muller S: The nucleolar SUMO-specific protease SENP3 reverses SUMO modification of nucleophosmin and is required for rRNA processing. EMBO Rep 9: 273-279, 2008.

56. Eifler K and Vertegaal AC: Mapping the SUMOylated landscape. FEBS J 282: 3669-3680, 2015.

57. Ma RG, Zhang Y, Sun TT and Cheng B: Epigenetic regulation by polycomb group complexes: Focus on roles of CBX proteins. J Zhejiang Univ Sci B 15: 412-428, 2014.

58. Li J, Xu Y, Long XD, Wang W, Jiao HK, Mei Z, Yin QQ, Ma LN, Zhou AW, Wang LS, et al: Cbx4 governs HIF-1 $\alpha$ to potentiate angiogenesis of hepatocellular carcinoma by its SUMO E3 ligase activity. Cancer Cell 25: 118-131, 2014.

59. Fukagawa A, Ishii H, Miyazawa K and Saitoh M: $\delta E F 1$ associates with DNMT1 and maintains DNA methylation of the E-cadherin promoter in breast cancer cells. Cancer Med 4: 125-135, 2015.

This work is licensed under a Creative Commons Attribution-NonCommercial-NoDerivatives 4.0 International (CC BY-NC-ND 4.0) License. 